Early Closure of a Temporary lleostomy in Patients With Rectal Cancer A Multicenter Randomized Controlled Trial

Danielsen, Anne K; Park, Jennifer; Jansen, Jens E; Bock, David; Skullman, Stefan; Wedin, Anette; Marinez, Adiela Correa; Haglind, Eva; Angenete, Eva; Rosenberg, Jacob

Published in:

Annals of Surgery

DOI:

10.1097/SLA.0000000000001829

Publication date:

2017

Document version

Publisher's PDF, also known as Version of record

Document license:

CC BY-NC-ND

Citation for published version (APA):

Danielsen, A. K., Park, J., Jansen, J. E., Bock, D., Skullman, S., Wedin, A., Marinez, A. C., Haglind, E.,

Angenete, E., \& Rosenberg, J. (2017). Early Closure of a Temporary lleostomy in Patients With Rectal Cancer: A Multicenter Randomized Controlled Trial. Annals of Surgery, 265(2), 284-290.

https://doi.org/10.1097/SLA.0000000000001829 


\title{
Early Closure of a Temporary lleostomy in Patients With Rectal Cancer
}

\author{
A Multicenter Randomized Controlled Trial
}

\author{
Anne K. Danielsen, PhD, MA(Ed), MA(ClN), RN, ${ }^{*}$ Jennifer Park, MD, $\dagger$ Jens E. Jansen, MD, $\ddagger$ \\ David Bock, PhD, $\dagger$ Stefan Skullman, MD, PhD, $\S$ Anette Wedin, RN, $\dagger$ Adiela Correa Marinez, MD, $\dagger$ \\ Eva Haglind, $M D, P h D, \dagger$ Eva Angenete, $M D, P h D, \dagger$ and Jacob Rosenberg, MD, DSc*
}

\begin{abstract}
Objective: The objective was to study morbidity and mortality associated with early closure (8-13 days) of a temporary stoma compared with standard procedure (closure after $>12$ weeks) after rectal resection for cancer.

Background: A temporary ileostomy may reduce the risk of pelvic sepsis after anastomotic dehiscence. However, the temporary ileostomy is afflicted with complications and requires a second surgical procedure (closure) with its own complications. Early closure of the temporary ileostomy could reduce complications for rectal cancer patients.

Methods: Early closure (8-13 days after stoma creation) of a temporary ileostomy was compared with late closure $(>12$ weeks) in a multicenter randomized controlled trial, EASY (www.clinicaltrials.gov, NCT01287637) including patients undergoing rectal resection for cancer. Patients with a temporary ileostomy without signs of postoperative complications were randomized to closure at 8 to 13 days or late closure $(>12$ weeks after index surgery). Clinical data were collected up to 12 months. Complications were registered according to the Clavien-Dindo Classification of Surgical Complications, and Comprehensive Complication Index was calculated.

Results: The trial included 127 patients in eight Danish and Swedish surgical departments, and 112 patients were available for analysis. The mean number of complications after index surgery up to 12 months follow up was significantly lower in the intervention group (1.2) compared with the control group (2.9), $P<0.0001$.
\end{abstract}

Conclusions: It is safe to close a temporary ileostomy 8 to 13 days after rectal resection and anastomosis for rectal cancer in selected patients without clinical or radiological signs of anastomotic leakage.

From the *Department of Gatroenterology, Herlev and Gentofte Hospital, University of Copenhagen, Herlev Ringvej, Herlev, Denmark; †Department of Surgery, Institute of Clinical Sciences, Sahlgrenska Academy, University of Gothenburg, Scandinavian Surgical Outcomes Research Group, Sahlgrenska University Hospital, Östra, Gothenburg, Sweden; $\ddagger$ Department of Surgery, Nordsjællands Hospital, Denmark; and §Department of Surgery, Skaraborgs Sjukhus Skovde, Sweden.

Disclosure: A.K.D. has received funding from the Research Council at Herlev Hospital, Denmark. S.S. received funding from the Health and Medical Care Committee of the Regional Executive Board and Region Västra Götaland (Nr. 311031, 390991), and from Lions Cancerfond West, Sweden. E.A. has received funding from the Swedish Research Council 2012-1786, the Swedish Cancer Society 2013/500, Sahlgrenska University Hospital, Agreement concerning research and education of doctors ALFGBG-366481, ALFGBG526501, and ALFGBG-493341, the Swedish Society of Medicine SLS247661 and SLS-412151, the Lions Väst Cancer Foundation.

The authors declare no conflicts of interest.

This is an open-access article distributed under the terms of the Creative Commons Attribution-Non Commercial-No Derivatives License 4.0, where it is permissible to download and share the work provided it is properly cited. The work cannot be changed in any way or used commercially.

Reprints: Anne K. Danielsen, PhD, MA(Ed), MA(CIN), RN, Department of Gastroenterology, Herlev Hospital, University of Copenhagen, Herlev Ringvej, Herlev, Denmark. E-mail: anne.k.danielsen@gmail.com.

Copyright (C) 2016 Wolters Kluwer Health, Inc. All rights reserved.

ISSN: 0003-4932/16/26502-0284

DOI: $10.1097 /$ SLA.0000000000001829
Keywords: Clavien-Dindo classification, early closure, ileostomy, morbidity, mortality, randomized controlled trial

(Ann Surg 2017;265:284-290)

$P$ atients with low rectal cancer often receive a temporary ileostomy at the time of resection of the rectum to reduce the risk of symptomatic anastomotic dehiscence. ${ }^{1}$ The temporary ileostomy was introduced to decrease the clinical consequences of an anastomotic leak but it has not been shown to reduce the risk of anastomotic leakage as such. ${ }^{2}$ However, studies have shown complication rates up to $43 \%$ related to the temporary ileostomy, ${ }^{3}$ including readmissions, dehydration, and chronic renal failure. ${ }^{4}$ Most patients with a temporary ileostomy will keep their stoma at least 3 months, and it is not unusual that the stoma is left in place much longer, and for a few patients it becomes permanent. ${ }^{5}$

Closure of the temporary ileostomy is associated with a low mortality, ${ }^{6}$ but the morbidity may be more than $20 \%{ }^{3}$ Timing of stoma closure has previously been investigated in a few prospective studies that mainly focused on morbidity and mortality related to early closure of the stoma. ${ }^{7-9}$ These studies did not demonstrate any significant effect on morbidity or mortality related to early closure. In other studies investigating the possibility of an earlier closure, the results have been promising. ${ }^{10,11}$

The aim of this trial was to explore if morbidity and mortality was reduced within 12 months after rectal resection in patients treated for low rectal cancer, and if the stoma was closed early (8-13 days after stoma creation) compared with late closure of the temporary ileostomy (a minimum of 12 weeks after stoma creation). The primary endpoint was the mean number of complications after index-operation and up to 12 months. Two secondary endpoints were (i) the proportion of patients with at least one complication with Clavien-Dindo Classification of Surgical Complications (ClavienDindo classification) ${ }^{12,13}$ severity grade IIIa, IIIb, IVa, IVb, or V after index surgery and up to 12 months and (ii) the mean number of stoma-related complications after index surgery and up to 12 months. Supporting endpoints were the Comprehensive Complication Index $(\mathrm{CCI})^{14}$ after index surgery and up to 12 months and creatinine levels.

\section{METHODS}

\section{Design}

The trial was designed as a randomized controlled multicenter trial comparing early with late closure of temporary ileostomy in eight Danish and Swedish surgical departments. Screening for and inclusion of participants was made after the index surgery (total mesorectal excision (TME) for rectal cancer) with creation of a temporary ileostomy. Follow-up of patients was at the time of 
closure, and 3, 6, and 12 months after index surgery. ${ }^{15}$ The study was designed and performed within the framework of the Scandinavian Surgical Outcomes Research Group (http://www.ssorg.net). The protocol for the EASY-trial (Early closure of a temporary ileostomy) was published at initiation of the study. ${ }^{15}$

\section{Participants}

After the index operation and before inclusion, consenting patients were clinically assessed by a surgeon on day 1 to 4 after index surgery to ensure that there were no clinical signs of postoperative complications such as infections or clinical signs of leakage. Patients without any adverse signs were invited to participate and after informed consent went through further investigation with a contrast computed tomography scan (CT-scan) or a flexible endoscopy of the rectum or both. This was performed 6 to 8 days after stoma creation to make sure that no patients with signs of anastomotic leakage were included. The CT-scan was performed with water soluble contrast through a catheter placed in the rectum, the rectoscopy was performed using either a flexible or rigid rectoscope. Both procedures were done without general anesthesia. Further details of the procedure for the clinical assessment and the CT-scan have been described elsewhere. ${ }^{15}$ The full list of criteria for inclusion and exclusion of patients is accessible in the research protocol. ${ }^{15}$ All eligible participants were registered in a screening$\log$ at the involved centers, reporting reasons for noninclusion.

\section{Randomization}

Consenting patients who fulfilled the inclusion criteria were randomized either to the intervention group with early closure (day 8-13 after stoma creation) or to the control group with late closure ( $>12$ weeks after stoma creation) of the ileostomy. Randomization was done in computer-generated blocks of six. The randomization was performed in the surgical ward using sequentially numbered thick, opaque, sealed envelopes. Blinding of the intervention was not possible.

\section{Procedures}

The surgical technique for closure was according to the local standard procedure and left to the discretion of the surgeon.

Clavien-Dindo classification was used to evaluate postoperative complications. ${ }^{12,13}$ The classification of each registered complication was made at the participating hospitals, and the clinical report forms (CRFs) included the full definition of the grades in the Clavien-Dindo system. Classification was summarized at the individual level by the CCI. ${ }^{14,16}$

\section{Outcomes}

The primary endpoint was the mean number of complications after the index-operation and up to 12 months. Five CRFs were collected at baseline, at closure, and at 3, 6, and 12 months. All complications within 12 months postoperatively were registered. The stoma related complications were registered retrospectively by inspection of patient charts filled in by stoma care therapists during hospital stay and in the outpatient clinics. Creatinine levels were measured before index surgery and at closure of the ileostomy. Other variables recorded prospectively in the CRFs were blood loss, American Society of Anesthesiologist's classification (ASA), duration of surgery, height of tumor localization, level of anastomosis, adjuvant oncological treatment, length of hospital stay, and complications, endoscopic or radiologic assessment of the anastomosis, reoperation, or readmission. The operating surgeon was also given an open-ended question in the operative-CRF to comment on surgical details.

The coding of the complications was done by research nurses, by the surgeon discharging the patient, or by the surgeon operating the patient. The codings were afterwards examined by one of the authors (J.P.) not involved in the operation, in order to ensure that no complications were coded twice, and that the coding was correct for each complication.

All eligible participants were registered in a screening-log in the involved centers, reporting reasons for noninclusion. All data were sent to the trial secretariat and entered into the trial database.

\section{Sample Size}

The study protocol specified 72 evaluable patients per group to have $80 \%$ power to detect a $66 \%$ reduction in the risk of complications. ${ }^{15}$ Because of slow enrollment, fewer evaluable patients were reached and before data analysis a re-calculation was performed. With 60 evaluable patients per group we would have $80 \%$ power to detect a $62.5 \%$ reduction in the annual mean number of complications using a 2-sided test with $5 \%$ significance level and assuming a mean complication rate of 0.45 in the control group.

\section{Statistical Methods}

A detailed statistical analysis plan was made before data analysis. Analyses were made on all randomized patients using an intention to treat perspective (ITT). All primary and secondary endpoints were analyzed with a generalized linear model with a log-link function with group (early vs late reversal) and hospital as factors. ${ }^{17}$ For the primary endpoint a negative binomial distribution was used. Results were to be presented as geometric mean ratio, $95 \%$ confidence intervals (CI) and a $P$-value for the test of a null hypothesis of no difference in average number of complications up to 12 months post index-surgery. For the first secondary endpoint a binomial distribution was used with robust variance estimation using Generalized Estimating Equations. Results are presented as risk ratio and $95 \% \mathrm{CI}$. For the second secondary endpoint a negative binomial distribution was used. The familywise error rate was controlled in the strong sense by a fixed sequence Bonferroni procedure where the two secondary hypotheses were only tested at $2.5 \%$ level if the primary hypothesis was rejected at the $5 \%$ level. ${ }^{18}$ Sensitivity adjusted analyses were performed with sex, age, body mass index (BMI), comorbidity, and radiotherapy as covariates.

\section{Ethics and Informed Consent}

The project was approved by the Science Ethical Committee for the Capital Region in Denmark (Protocol ID: H-1-2010-113) and in Sweden the project was approved by the Ethical Approval Committee in Göteborg (Dnr 064-2011). Before inclusion patients were informed about the study and all participating patients returned a signed consent form.

\section{Approval of Data Security}

The project was approved by the Data Protection Agency in Denmark, and by the Personal Data Representative at the Sahlgrenska University Hospital, Göteborg, Sweden.

\section{Trial Registration}

The project was registered at www.clinicaltrials.gov (identifier: NCT01287637) before patient inclusion.

\section{RESULTS}

\section{Overall Results}

We assessed 418 patients for eligibility, and 291 of these did not meet the inclusion criteria because of suspected anastomotic leakage $(n=37)$, unwillingness to participate $(n=53)$, other medical reasons $(n=159)$, and where inclusion was missed $(n=42)$ (Fig. 1). Hence, 127 patients were randomized and out of these another 


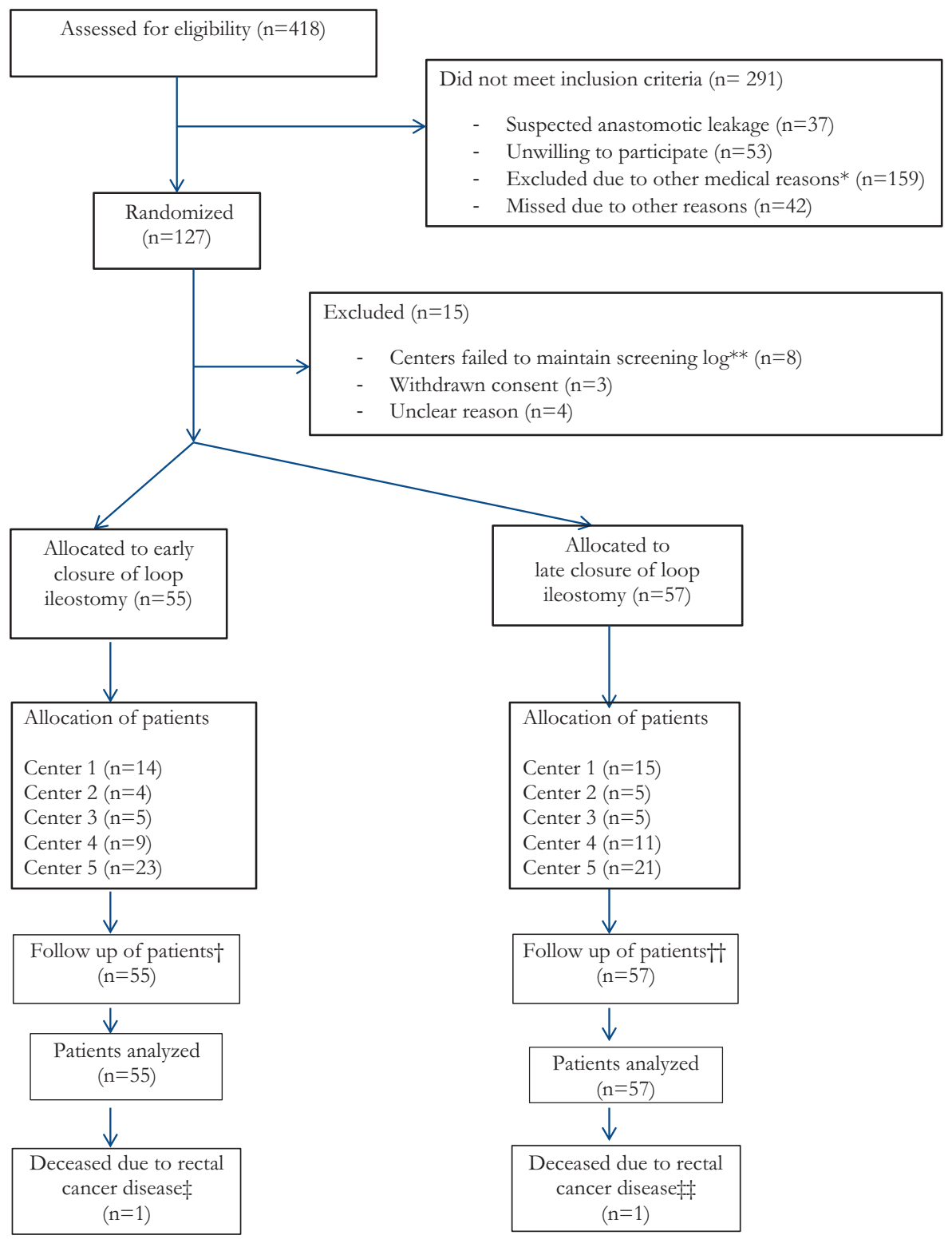

* excluded after clinical evaluation $(n=91)$, diabetes $(n=28)$, received permanent stoma or no stoma $(n=29)$, steroid treatment $(n=3)$, other $(n=8)$.

** center $6(n=2)$, center $7(n=3)$ and center $8(n=3)$

+ missing follow up at 3 months: $(n=1)$, at 12 months $(n=2)$

it missing follow up at closure: $(n=1), 3$ months: $(n=2), 6$ months: $(n=3), 12$ months: $(n=2)$

t missing follow up at 12 months

It patient did not go through closure, missing follow up at 12 months

FIGURE 1. Participant flow diagram.

15 patients were excluded. In summary, 112 patients were included from February 2011 and with last follow-up of the last patient in November 2015. There were 55 patients in the early- and 57 patients in the late closure group available for analysis, respectively (Fig. 1). There were no violations of the randomization.

Baseline demographic data are shown in Table 1. The intervention group included a larger proportion of females compared with the control group (Table 1). In all other respects the groups were comparable. Perioperative details of loop ileostomy closure and its postoperative complications were comparable between groups (Tables 2 and 3).

Median time from index surgery to closure was 11 days in the early group and 148 days in the late group (Table 2). At 3, 6, and 12-months follow up after index surgery there were more 
TABLE 1. Baseline and Preoperative Characteristics of Included Patients

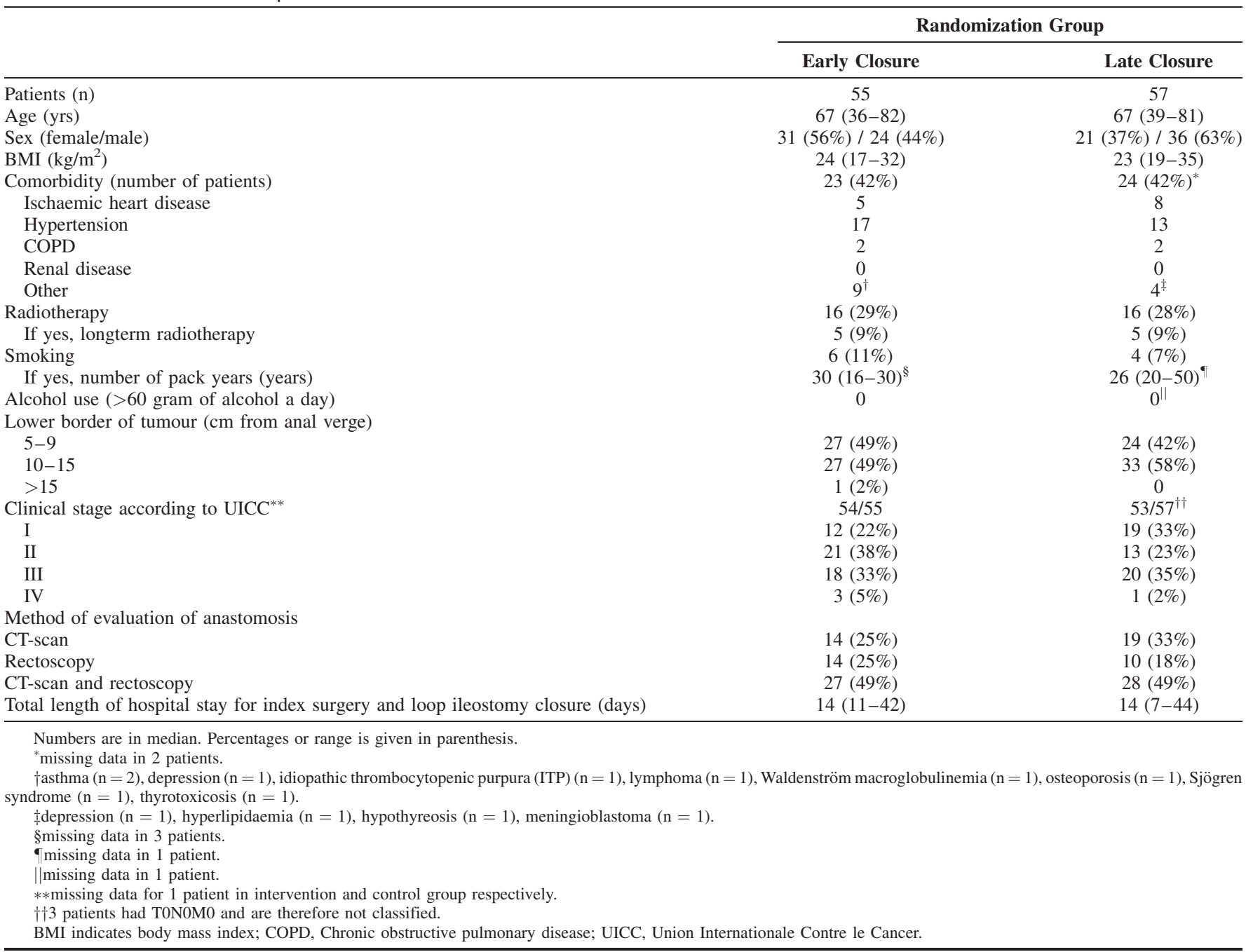

complications in the control group (Table 3). Two patients in the control group underwent earlier closure because of high-volume stomal output and stomal retraction, respectively. These events were regarded as complications classified as Clavien-Dindo Grade IIIb (Table 3). One patient in the intervention group suffered from severe renal failure because of high volume output at the 3 months follow up (classified Clavien-Dindo Grade IVa). This patient had undergone a failed attempt to closure within 13 days of index surgery, which lead to prolonged duration of the loop ileostomy. The attempted surgery was considered a postoperative complication, and the patient remained in the early closure group on an intention to treat basis.

Primary endpoint: The mean number of complications after index surgery up to the 12 months follow up was significantly lower in the intervention group (mean number of complications was 1.24 in the intervention group compared with 2.88 in the control group) with a ratio for intervention versus control of 0.42 (95\% CI $0.32-0.57), P<$ 0.0001 (Tables 2 and 3 ). The adjusted analysis showed similar results.

\section{Secondary Endpoints}

When comparing more severe complications (Clavien-Dindo Grade IIIa or higher), there was no significant difference between the two groups (proportion of patients with at least one complication
Clavien-Dindo Grade IIIa or higher was 0.22 for the intervention group and 0.29 for the control group, $P=0.32$ ). The results were similar in the adjusted analysis.

The mean number of stoma related complications was significantly higher in the control group (intervention group 0.30 and control group 1.25) with a ratio for intervention versus control of 0.25 (95\% CI $0.15-0.42), P<0.0001$ (Table 4). The adjusted analysis showed similar results.

\section{Supporting Endpoints}

There was a difference of 15.7 units in median level of CCI (8.7 and 24.4 for early and late group, respectively).

\section{Creatinine Levels}

The mean levels at baseline were 72.7 and $75.8 \mathrm{micromol} / \mathrm{L}$ with average intraindividual changes to closure of 0.40 and 9.20 for early and late group, respectively.

\section{DISCUSSION}

This clinical trial provides evidence of the safety, efficacy, and feasibility of early closure of a temporary ileostomy in selected 
TABLE 2. Details of Loop lleostomy Closure

\begin{tabular}{|c|c|c|}
\hline & \multicolumn{2}{|c|}{ Randomization Group } \\
\hline & Early Closure $(\mathbf{n}=\mathbf{5 5})$ & Late Closure $(\mathbf{n}=\mathbf{5 7}) *$ \\
\hline Time with ileostomy (days) & $11(8-152)^{\dagger}$ & $148(64-665)^{\ddagger}$ \\
\hline Time duration of surgery (min) & $50(17-180)$ & $71(31-401)$ \\
\hline \multicolumn{3}{|l|}{ Anastomosis (ileo-ileal anastomosis) } \\
\hline Handsewn & $45(82 \%)$ & $40(70 \%)$ \\
\hline Stapled & $10(18 \%)$ & $15(26 \%)$ \\
\hline Time until fully oral nutrition (days) & $1(0-23)$ & $2(0-20)$ \\
\hline Time in intensive care unit (days) & $0(0-0)$ & $0(0-0)$ \\
\hline Hospital stay after closure (days) & $4(2-27)$ & $4(2-28)$ \\
\hline Postoperative complication (number of patients) & $4(7 \%)$ & $4(7 \%)^{\S}$ \\
\hline \multicolumn{3}{|c|}{ Classification according to Clavien-Dindo ${ }^{12,13}$ (number of complications) } \\
\hline Grade I & 1 & 0 \\
\hline Grade II & 3 & 1 \\
\hline Grade V & 0 & 0 \\
\hline \multicolumn{3}{|l|}{ Type of complication } \\
\hline Infection & 2 & 0 \\
\hline Fistula/anastomotic leakage & 0 & 1 \\
\hline Bleeding & 0 & 0 \\
\hline Nausea/vomiting & 1 & 2 \\
\hline Cardiopulmonary & 0 & 0 \\
\hline Liver insufficiency & 0 & 0 \\
\hline Pain & 1 & 1 \\
\hline Allergy & 0 & 0 \\
\hline Pancreatitis & 0 & 0 \\
\hline Other (specification) & $2^{\circ}$ & $1^{\|}$ \\
\hline \multicolumn{3}{|l|}{ Cause of reoperation - after loop ileostomy closure } \\
\hline Failed attempt of stoma closure & 1 & \\
\hline
\end{tabular}

patients with rectal cancer with a follow up of 12 months after index operation. Only patients assessed for clinically relevant complications related to the index surgery and who were found appropriately fit were invited to enter the trial. Therefore, only patients not showing clinical or radiological signs of adverse events after the rectal cancer operation were included and randomized.

We found a significant difference between the two groups regarding our primary endpoint, which was the mean number of complications within 12 months of index surgery. Further, patients in the intervention group had fewer complications than patients in the control group during the follow up. There was no significant difference in the rate of severe complications, grade IIIa, and above according to the Clavien-Dindo classification. The pattern of severe complications $>$ CD IIIa varied between intervention and control groups during the follow-up period (Table 3 ) and in particular there seemed to be more severe complications in the control group between 6 and 12 months compared with the intervention group. The total number of complications differed significantly over time between groups. In the study by Alves et al, ${ }^{9}$ patients had an overall morbidity of $31 \%$ in the early closure group and $38 \%$ in the late closure group at 90 days after stoma creation, which was similar to our results. However, they did not report overall complications later in the follow-up period.

Patients included in this trial were comparable with regard to baseline characteristics apart from more female participants in the intervention group. The adjusted analysis showed similar results with no impact of sex, age, BMI, comorbidity, or neoadjuvant radiotherapy.

Data on surgical closure of the loop ileostomy did not differ between the groups, although 3 patients in the intervention group and 4 patients in the control group experienced complications and some of them had more than one complication. Our results indicated that early closure of a temporary ileostomy is safe, as has been suggested before. ${ }^{6}$ The rate of overall complications reported from other studies was higher ${ }^{9,19,20}$ and one possible explanation could be criteria for inclusion and exclusion in our trial.

We found a significant difference in the mean number of stoma related complications, in particular relieving patients in the 
TABLE 3. Details of Complications Registered at Follow-up 3, 6, and 12 Months After Index Operation and Including Complications Occurring From Leaving Hospital Until 3 Months, From 3 to 6 Months and From 6 to 12 Months, Respectively

\begin{tabular}{|c|c|c|}
\hline \multirow[b]{2}{*}{ Classification According to Clavien-Dindo ${ }^{12,13}$ (Number of Complications) } & \multicolumn{2}{|c|}{ Randomization Group } \\
\hline & Early Closure $(n=55)$ & Late Closure $(n=57)$ \\
\hline 3 months & missing $\mathrm{n}=1$ & missing $\mathrm{n}=2$ \\
\hline Postoperative complication (number of patients) & $18 / 55(33 \%)$ & $26 / 57(46 \%)$ \\
\hline Grade I & 7 & 11 \\
\hline Grade II & 4 & 11 \\
\hline Grade IIIa & 5 & 9 \\
\hline Grade IIIb & 4 & 2 \\
\hline Grade IVa & 1 & 0 \\
\hline Grade IVb & 0 & 0 \\
\hline Grade V & 0 & 0 \\
\hline 6 months & & missing $\mathrm{n}=3$ \\
\hline Postoperative complication (number of patients) & $4 / 55(7 \%)$ & $18 / 57(32 \%)$ \\
\hline Grade I & 2 & 14 \\
\hline Grade II & 3 & 7 \\
\hline Grade IIIa & 0 & 3 \\
\hline Grade IIIb & 0 & 0 \\
\hline Grade IVa & 0 & 0 \\
\hline Grade IVb & 0 & 0 \\
\hline Grade V & 0 & 0 \\
\hline 12 months & missing $\mathrm{n}=2$ & $\operatorname{missing} \mathrm{n}=2$ \\
\hline Postoperative complication (number of patients) & $8 / 55(15 \%)$ & $19 / 57(33 \%)$ \\
\hline Grade I & 5 & 6 \\
\hline Grade II & 2 & 6 \\
\hline Grade IIIa & 1 & 4 \\
\hline Grade IIIb & 1 & 2 \\
\hline Grade IVa & 0 & 2 \\
\hline Grade IVb & 0 & 0 \\
\hline Grade V & 0 & 0 \\
\hline Reoperations within 12 months - causes & $5 / 55(9 \%)$ & $4 / 57(7 \%)$ \\
\hline Small bowel obstruction & 1 & 1 \\
\hline Presacral abscess (leakage of the colo-anal anastomosis) & 1 & 2 \\
\hline Abscess & 2 & 0 \\
\hline Bleeding peptic ulcer & 1 & 0 \\
\hline Stenosis in colo-anal anastomosis & 0 & 1 \\
\hline
\end{tabular}

TABLE 4. Details of Complications Related to the Loop lleostomy From Index Surgery Until Closure of the Stoma

\begin{tabular}{|c|c|c|}
\hline \multirow[b]{2}{*}{ Classification According to Clavien-Dindo ${ }^{12,13}$ (Number of Complications) } & \multicolumn{2}{|c|}{ Randomization Group } \\
\hline & Early Closure $(n=55)$ & Late Closure $(n=57)$ \\
\hline \multicolumn{3}{|l|}{ Stoma related complications } \\
\hline Number of patients & $13 / 55(24 \%)$ & $44 / 57(77 \%)$ \\
\hline Grade I & 13 & 63 \\
\hline Grade II & 3 & 3 \\
\hline Grade IIIa & 0 & 2 \\
\hline Grade IIIb & 0 & $3^{\dagger}$ \\
\hline Grade IVa & $1^{*}$ & 0 \\
\hline Grade IVb & 0 & 0 \\
\hline Grade V & 0 & 0 \\
\hline \multicolumn{3}{|l|}{ Type of stoma related complications } \\
\hline Skin irritation & 3 & 16 \\
\hline Stomal ulcer & 2 & 18 \\
\hline Parastomal infection & 1 & 0 \\
\hline Leakage outside appliance bag & 3 & 17 \\
\hline High volume output & 5 & 9 \\
\hline Parastomal hernia & 0 & 2 \\
\hline Stenosis & 2 & 2 \\
\hline Prolaps & 0 & 1 \\
\hline Retraction & 0 & 2 \\
\hline Other & 1 & 4 \\
\hline
\end{tabular}

${ }^{*}$ Renal failure becuase of high volume output. This patient underwent a failed attempt to closure within 13 days after index surgery, which lead to delayed loop ileostomy closure. $\dagger(\mathrm{i})$ Stomal retraction $(\mathrm{n}=1)$, which lead to earlier closure, (ii) symptomatic parastomal hernia $(\mathrm{n}=1)$, which was sutured at closure, and (iii) high volume output $(\mathrm{n}=1)$, which lead to earlier closure of the loop ileostomy. 
intervention group from skin irritation, ulcerations, and problems with leakage and of high-volume output. Although stoma related complications may seem less severe than complications $>$ IIIa in the Clavien-Dindo classification, these complications can be tiresome, distressing, and embarrassing for the patient. ${ }^{21,22}$ The observed difference in median CCI of 15.7 units pointed to a clinically relevant advantage for the intervention group. ${ }^{14}$

Many patients have their loop ileostomy considerably longer than the suggested 12 weeks, which also could be seen in our study where median time until closure in the control group was 148 days (equivalent to 5 months). Other studies have pointed out that $20 \%$ of the temporary ileostomies were never closed, ${ }^{23}$ but only 3 patients (3\%) in our trial did not undergo closure within the follow-up period of 12 months after index surgery. Of those, two patients did eventually undergo reversal of the loop ileostomy, although later than 12 months after index surgery, and the third patient who deceased from metastatic disease within 12 months was regarded as a permanent loop ileostomy carrier. Previous reports have suggested reasons for nonclosure as old age, surgical complications, and comorbidity. ${ }^{5}$ Possible explanations for the high closure rate $<12$ months in our trial most likely include focus on closure of the ileostomy in the trial setting.

In accordance with the report by Gessler et $\mathrm{al},{ }^{4}$ we found that a temporary ileostomy has a negative effect on renal function, reflecting that for a few individuals a loop ileostomy may have a systemic effect as well.

Strengths of this trial include the randomized design, the screening $\log$ at the including hospitals enabling an evaluation of external validity and the thorough examination of patients after inclusion and before randomization to ascertain that patients did not have a subclinical anastomotic leakage.

It could be considered a limitation that not all patients screened for participation were included because of the strict inclusion criteria. We screened 418 patients in the participating centers and only 127 patients were included and randomized and finally 112 patients were included in the analysis (Fig. 1). However, this was a safety measure and even if only $30 \%$ of the entire group can undergo early closure this represents significant improvements for the patients. The screening-logs were complete and all nonincluded patients were registered with specific reasons for the noninclusion. Another limitation was the coding of complications as it was not possible to blind the surgeon or the research nurse who carried out the actual coding of the complications, which may have led to observer bias.

\section{CONCLUSIONS}

We found that in selected patients without clinical, radiological, or endoscopic signs of a leakage early closure of the temporary ileostomy after surgery for rectal cancer resulted in a significantly lower mean number of complications compared with late closure. We also found low numbers of severe complications in both groups suggesting that patients should be considered for early closure of an ileostomy if they have no signs of anastomotic leakage in the postoperative period after rectal resection.

\section{ACKNOWLEDGMENTS}

The authors would like to thank doctors and nurses at the participating centres for their help and assistance in identifying and including patients and operating and caring for patients during the course of the trial.

\section{REFERENCES}

1. Matthiessen P, Hallböök $O$, Rutegård J, et al. Defunctioning stoma reduces symptomatic anastomotic leakage after low anterior resection of the rectum for cancer. Ann Surg. 2007;246:207-214.

2. Montedori A, Cirocchi R, Farinella E, et al. Covering ileo- or colostomy in anterior resection for rectal carcinoma. Cochrane Database Syst Rev. 2010. CD006878:1-22.

3. Gessler B, Haglind E, Angenete E. Loop ileostomies in colorectal cancer patients-morbidity and risk factors for nonreversal. J Surg Res. 2012; 178:708-714

4. Gessler B, Haglind E, Angenete E. A temporary loop ileostomy affects renal function. Int J Colorectal Dis. 2014;29:1131-1135.

5. den Dulk M, Smit M, Peeters KCMJ, et al. A multivariate analysis of limiting factors for stoma reversal in patients with rectal cancer entered into the total mesorectal excision (TME) trial: a retrospective study. Lancet Oncol. 2007;8:297-303.

6. Chow A, Tilney HS, Paraskeva P, et al. The morbidity surrounding reversal of defunctioning ileostomies: a systematic review of 48 studies including 6107 cases. Int J Colorectal Dis. 2009;24:711-723.

7. Menegaux F, Jordi-Galais P, Turrin N, et al. Closure of small bowel stomas on postoperative day 10. Eur J Surg. 2002;168:713-715.

8. Bakx R, Busch ORC, van Geldere D, et al. Feasibility of early closure of loop ileostomies: a pilot study. Dis Colon Rectum. 2003;46:1680-1684.

9. Alves A, Panis Y, Lelong B, et al. Randomized clinical trial of early versus delayed temporary stoma closure after proctectomy. Br J Surg. 2008;95:693-698.

10. Robertson JP, Puckett J, Vather R, et al. Early closure of temporary loop ileostomies: a systematic review. Ostomy Wound Manage. 2015;61:50-57.

11. Hindenburg T, Rosenberg J. Closing a temporary ileostomy within two weeks. Dan Med Bull. 2010;57:1-5.

12. Clavien PA, Barkun J, de Oliveira ML, et al. The Clavien-Dindo classification of surgical complications: five-year experience. Ann Surg. 2009;250:187-196.

13. Dindo D, Demartines N, Clavien P-A. Classification of surgical complications: a new proposal with evaluation in a cohort of 6336 patients and results of a survey. Ann Surg. 2004;240:205-213.

14. Slankamenac K, Nederlof N, Pessaux P, et al. The Comprehensive Complication Index: a novel and more sensitive endpoint for assessing outcome and reducing sample size in randomized controlled trials. Ann Surg. 2014; 260:757-763.

15. Danielsen AK, Correa-Marinez A, Angenete E, et al. Early closure of temporary ileostomy - the EASY trial: protocol for a randomised controlled trial. BMJ Open. 2011;1:1-7.

16. Slankamenac K, Graf R, Barkun J, et al. The Comprehensive Complication Index: a novel continuous scale to measure surgical morbidity. Ann Surg. 2013;258:1-7.

17. McCullagh P, Nelder J. Generalized Linear Models. 2nd ed. Chapman \& Hall/ CRC; 1989

18. Burman C-F, Sonesson C, Guilbaud O. A recycling framework for the construction of Bonferroni-based multiple tests. Stat Med. 2009;28:739-761.

19. Luglio G, Pendlimari R, Holubar SD, et al. Loop ileostomy reversal after colon and rectal surgery: a single institutional 5-year experience in 944 patients. Arch Surg. 2011;146:1191-1196.

20. van Westreenen HL, Visser A, Tanis PJ, et al. Morbidity related to defunctioning ileostomy closure after ileal pouch-anal anastomosis and low colonic anastomosis. Int J Colorectal Dis. 2012;27:49-54.

21. Robertson I, Leung E, Hughes D, et al. Prospective analysis of stoma-related complications. Color Dis. 2005;7:279-285.

22. Sun V, Grant M, McMullen CK, et al. Surviving colorectal cancer: long-term, persistent ostomy-specific concerns and adaptations. $J$ Wound Ostomy Continence Nurs. 2013;40:61-72.

23. David GG, Slavin JP, Willmott S, et al. Loop ileostomy following anterior resection: is it really temporary? Color Dis. 2010;12:428-432. 